\title{
Isolated ventricular septal defect in infants
}

\author{
Zeynep Eyileten $^{1}$, Adnan Uysalel ${ }^{1}$
}

1) Ankara University Faculty of Medicine, Department of Cardiovascular Surgery, Prof. Dr., Ankara, Turkey

\begin{abstract}
Ventricular septal defect is a hole in the interventricular septum and may be isolated or associated with other major defects involving patency of the arterial duct, coarctation of aorta, pulmonary stenosis, tetralogy of Fallot, double-outlet right ventricle, truncus arteriosus, and transposition, and account for over half of the patients with congenital heart disease. Isolated ventricular septal defect is the most commonly recognized congenital heart anomaly in children and accounts for about one-fifth of all congenital heart disease. In this chapter, we'll cover isolated ventricular septal defects.
\end{abstract}

Key words: Ventricular septal defect, isolated, infant. 


\section{Classification}

The interventricular septum (IVS) is commonly divided into four parts - the membranous septum and the inlet, trabecular, and outlet portions of the muscular septum. Depending on their location in the IVS, isolated ventricular septal defect (VSD) can be divided into four types; $;^{(1,2,3)}$

Type I VSDs (Doubly commited/ Subarterial/ Outlet/ Infundibular/ Conotruncal/ Conal/ Juxtaarterial/ Supracristal) account for 5\% to $10 \%$ of isolated VSDs. They are located below the semilunar valve and above the crista supraventricularis. The right aortic leaflet most commonly is sucked into the defect, resulting in aortic incompetence in $40 \%$ to $50 \%$ of conal defects. Atrioventricular conduction tissue is far away from the borders of this type of VSD.

Type II VSDs (Perimembranous/ Conoventricular) are the most common isolated VSDs, comprising about $70 \%$ to $80 \%$ in most series. These defects are located in the membranous septum and may extend into the inlet or outlet. They are usually bordered cranially by aortic valve and posteroinferiorly by anteroseptal commissure of the tricuspid valve and they can undergo partial or complete closure by apposition of the septal leaflet of the tricuspid valve, forming a tricuspid valve "pouch" or "aneurysm of the ventricular septum". Less commonly the noncoronary aortic leaflet may prolapse through the defect, resulting in decrease of the defect as well as progressive aortic incompetence. Atrioventricular conduction axis penetrates directly through the posteroinferior border of the defect. These type of VSDs can be associated with malalignment of the aortopulmonary septum, as in the setting of tetralogy of Fallot (anterior malalignment) or interrupted aortic arch (posterior malalignment). A Gerbode-type defect is a communication between the left ventricle and right atrium through the membranous IVS.

Type III VSDs (Atrioventricular canal/ Inlet type) account for about $5 \%$ of isolated defects. They are situated beneath the septal leaflet of the tricuspid valve, with the tricuspid valve annulus forming their posterior border. They can be associated with primum type atrial septal defects and left atrioventricular valve clefts. The conduction tissue is closely related to the posteroinferior border of the defect. They have a remarkably high prevalence in genetic conditions such as trisomy 18 and trisomy 21 .

Type IV VSDs (Muscular/Trabecular) account for $10 \%$ to $15 \%$ of isolated VSDs. They have a rim totally made up of muscle and are divided into midmuscular (most common), apical (trabecular), and anterior muscular defects. The presence of multiple muscular defects is known as the "swiss cheese" septum. The conduction tissue is generally remote from the edges of a muscular defect.

The defects in the neonate are devided into 3 subgroups depending on their size; $;^{(1,4)}$

- Small (restrictive) VSD is $<4 \mathrm{~mm}$ ( or less than the $33 \%$ of cross-sectional diameter of the aortic root in catheterization), the pulmonary to systemic blood flow ratio (Qp:Qs) $<1.5$.

- Moderate VSD is 4-6 mm (or between the 33-75\% of cross-sectional diameter of the aortic root in catheterization), Qp:Qs $=1.5$ - 2.3.

- Large (non-restrictive) VSD is $>6 \mathrm{~mm}$ ( or larger than the $75 \%$ of cross-sectional diameter of the aortic root in catheterization), Qp:Qs $>2.3$.

\section{Patophysiology}

In non-restrictive VSDs, the magnitude of the shunt depends on the relative pulmonary (PVR) and systemic vascular resistance (SVR). Since in a normal individual SVR is greater than PVR, in smaller defects, the blood flow is from the left to right ventricle. In patients with a large VSD, the systolic pressure in both ventricles is the same, with right sided pressures elevated to the same levels as those normally present on the left side of the heart. Large VSDs place two major hemodynamic loads upon the ventricles; increased pressure load on the right ventricle and increased volume load on the left ventricle.

PVR is high at birth and little flow across the defect may exist during this period. When PVR shows a sharp fall by 2-8 weeks of age, in patients with large VSD, pulmonary blood flow increases secondary to increased left-to-right shunt. The augmented blood flow returns through the left atrium to the left ventricle. To acco- 
modate the increased pulmonary venous return, the left ventricle dilates. If the left ventricle becomes greatly dilated, the myocardium can not develop sufficient tension to maintainn the pressure volume relationship, causing congestive cardiac failure. Patients with large VSD, develop congestive heart failure and its symptoms of tachypnea, slow weight gain and poor feeding by 2 to 3 months of age.

As pulmonary vascular disease develops, PVR increases and left to right shunt reverses and the presence of a right to left shunt will result in cyanosis (Eisenmenger syndrome). Congestive heart failure lessens. These rarely present in children below age 5 with isolated VSDs and is usually seen in adolescents and adults. In a patient with high fixed PVR, the defect should not be closed. ${ }^{(1,2,3)}$

\section{Diagnosis}

Patients with small VSDs usually are asymptomatic. A loud holosystolic murmur may be heard best at the left sternal border. Pulmonary arterial pressure and pulmonary vascular resistance is near normal. The normal decline in PVR can be delayed in infants with moderate and large VSDs. Thus, murmurs may not be detected in such infants until several weeks postnatally.

Infants with moderate or large VSDs may present with pallor, tachypnea, increased work of breathing, poor weight gain, or failure to thrive, and diaphoresis particularly with feeding at approximately two to eight weeks of age. Right ventricular impulse, felt at the lower left sternal border is prominent. A precordial trill may be palpable. As the degree of pulmonary hypertension increases, the intensity of the pulmonary component of S2 will increase. There will be holosystolic, midfrequency murmur. In patients with large VSDs, in addition to the systolic murmur, there will be a middiastolic mitral flow murmur as a result of increased volume of blood flowing from the left atrium to the left ventricle. As the PVR rises, the holosystolic murmur may disappear entirely, leaving a loud second heart sound due to closure of the pulmonary valve which is indicative of severe pulmonary hypertension.

The electrocardiogram; may be normal in small
VSDs. Moderate sized VSD's may be commonly associated with hypertrophy of right, left or both ventricle. In patients with large VSDs, left atrial dilatation, manifested by biphasic P waves in leads I, AVR, and V6 associated with right ventricular hypertrophy and left or right axis deviation may be seen. Patients with pulmonary hypertension, will exhibit evidence of right ventricular hypertrophy in electrocardiography

In chest radiography; patients with small defects often will have a normal cardiac silhoutte and normal pulmonary markingsy, while moderate and large VSD patients will have increased pulmonary vascularity and cardiomegaly with biventricular enlargement. Lateral projections may show upward deviation of the left main bronchus due to left atrial dilatation in large defects. In patients with Eisenmenger syndrome, the heart may not be enlarged and the pulmonary vascular markings may not be increased. Only the main pulmonary artery is enlarged.

Transthoracic echocardiography is the mainstay for the definitive diagnosis of VSD. It allows delineation of the anatomic site of the VSD, associated cardiac lesions, pulmonary artery pressure, degree of cardiac dilatation. Cardiac catheterization is only indicated to determine; quantification of the ratio of systemic and pulmonary flows, the pulmonary artery pressure and accurate identification of smaller defects. ${ }^{(1)}$

\section{Natural history}

The incidence of spontaneous closure of VSDs is highest during the first year of life (40\%) but continues to a lesser degree up to about 5 years, after which spontaneous closure is rare. Most VSDs are restrictive and frequently become smaller or undergo spontaneous closure. Defects located in the muscular septum close with the growth and hypertrophy of the surrounding muscular septum. Small membranous defects may close through apposition of the septal leaflet of the tricuspid valve secondary to negative pressure created by the jet through the defect.

In patients with nonrestrictive VSD, congestive heart failure symptoms develop soon after birth, concomitant with the fall in the elevated neonatal pulmo- 
nary resistance. Patients are at risk of developing irreversible pulmonary vascular disease after 1-2 years of age (earlier in children with trisomy 21). Generally, outcome is good in patients of all ages when preoperative PVR is only mildly or moderately elevated. Large VSDs in such children should be repaired by three to four months of age. Eisenmenger syndrome, appearing most frequently in the second and third decade of life and typically leading to death by the age of 40 .

Some patients with isolated VSD develop subpulmonary stenosis due to right ventricular infundibular hypertrophy. These patients are not at risk of pulmonary vascular destructive disease. Prolapse of the leaflets of the aortic valve occurs most frequently with the defects opening directly into the outlet of the right ventricle, muscular outlet, or perimembranous defects opening to the right ventricular outlet with malalignment of the muscular outlet septum. Untreated prolapse of the aortic valvar leaflets results first, in a decreased left-toright shunt because the involved leaflet often prolapses into the defect and reduces the shunt, and second, worsening aortic insufficiency. ${ }^{(2,3)}$

\section{Indication for VSD repair}

- Large VSDs presenting in the first few months of life with severe congestive heart failure or inlet (AV canal) and outlet (supracristal) defects which do not generally close spontaneously are the VSD's in which surgical closure is indicated.

- An undesirable mechanism of spontaneous occlusion is prolapse of an aortic valvar leaflet into the defect, often causing aortic insuffiency. This is also an indication for surgical repair. It is preferable to identify the defects associated with this potential complication and close them before its development.

- Bacterial endocarditis is a rare complication. Following antibiotic treatment of the active infection, closure of VSD regardless of its size is indicated.

- All VSDs irrespective of size, if associated with another reason for cardiac surgery,

- All residual VSDs $>3 \mathrm{~mm}$ or those associated with elevated pulmonary artery pressures have also indication for closure. ${ }^{(2,5,6)}$
Early repair of congenital cardiac lesions with improved postoperative care is the best strategy to prevent the development of severe pulmonary vasculopathy in CHD. However, even in developed nations, some infants/children are older at presentation or have yetundiscovered genetic abnormalities that predispose to increased pulmonary vasoreactivity or early development of pulmonary vascular remodeling. The decision to operate on these patients with an acceptable risk of early and late postoperative complications is not easy, and the current view is that it should be not be based on single parameters.

During the 5th World Symposium on Pulmonary Hypertension of the World Health Organization (Nice, France, February 2013), a PVR of 4Wood units $\mathrm{m} 2$ and PVR:SVR ratio $<0.3$ was proposed as a limit for considering surgery, and a PVR of 4-8 Wood units $\mathrm{m} 2$ as the range in which patients should be discussed case by case. The PVR of 6 Wood units $\mathrm{m} 2$ and a PVR:SVR ratio of 0.3 as limits for considering operation in PAH-CHD was proposed and a $20 \%$ decrease in PVR from baseline during the acute vasodilator test is considered sufficient to define a positive response but not to characterize operability. There has been debate about what to do with patients with elevated PVR (e.g., PVR $>8-10$ Wood units $\mathrm{m} 2$ and PVR:SVR $>0.5$ ), in particular since the answer will not be the same for patients at different ages..$^{(7,8,9)}$

\section{Technique of surgical repairing of VSD}

The progress in cardiopulmonary bypass, myocardial protection, improved skill, and surgical techniques and in the perioperative care has advanced so that standard corrective operation for ventricular septal defect closure in infant patients is now obtained with almost no mortality or major morbidity. Today pulmonary artery banding is not preferred except for only a very few specific lesions like multiple muscular (Swiss cheese) VSDs, multiple VSDs with coarctation, single ventricle with large VSD, for preparing left ventricle in late (after 6 weeks of age) presented transposition of great arteries (TGA) and for training left ventricle for a double switch in congenitally corrected transposition of great arteries.

The corrective operative approach varies according 
to the type of VSD. Perimembranous and inlet VSDs are usually repaired through a right atrial approach. Doubly commited VSD's are approached through the pulmonary artery, aorta or right ventricle. Muscular VSDs are usually approached through right atrium or through limited right or left ventriculotomy.

Following aorting and bicaval cannulation, moderate hypothermia $\left(28-32 \mathrm{C}^{\circ}\right)$ is constituted. The aorta is clamped and the heart is arrested with cardioplegic perfusate. The right atrium is opened to expose the tricuspid valve orifice. The pump sump sucker is placed in the left ventricle through interatrial septum to keep the operative field dry. If atriotomy is not required, the sucker is placed via the right superior pulmonary vein or left atrial appendage.

The perimembranous VSD is identified by retracting the septal and anterior leaflets of tricuspid valve. In some instances, the VSD perimeter cannot be completely identified because of the overlying tricuspid valve tissue (tricuspid valve pouch). Tricuspid valve detachment may be required by incision on the septal leaflet, parallel to the tricuspid valve annulus. The next step is to inspect the VSD. The aortic leaflets may prolapsed into the defect and must be avoided during suture placement.

The technique of VSD closure that we have been using at Ankara University, Department of Cardiovascular Surgery, is to encircle the perimeter of the VSD with

\section{Figure 1. Dacron patch repair with interrupted sutures}

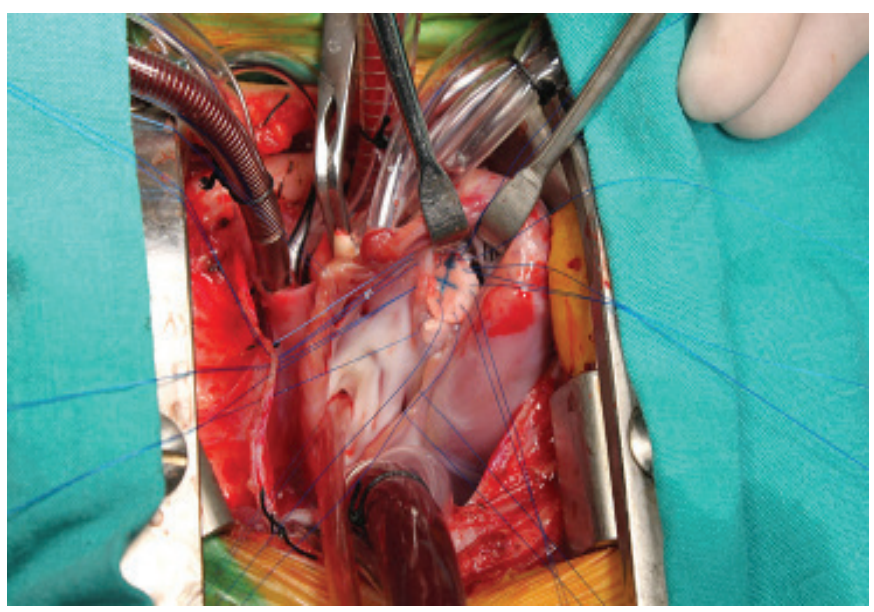

multiple, interrupted, pledget-based 5-0 polypropylene sutures (Figure-1). To avoid injury to the conducting system, the sutures should be placed superficially and carefully along the inferior and posterior margins of the defect and stay on the right ventricular side of the VSD. This closure begins from the area of the insertion of the muscle of Lancisi (medial papillary muscle of the conus) to the annulus of the tricuspid valve near the region of yhe triangle of Koch. Care must be taken when placing these sutures to avoid the aortic valve cusp.

The sutures are then sequentially placed through an appropriately sized patch (1.5 times the size of the actual hole in the septum), the patch is lowered into the defect, and the sutures are tied and cut. We commonly use Dacron patch. Alternatively, autologous pericardium, or polytetrafluoroethylene (Gore-Tex) patch material may be used. After the patch has been anchored by tying all the sutures, the tricuspid valve is repaired (usually with 6-0 prolene) if detachment was performed. The right ventricle can be irrigated with saline to identify tricuspid valve regurgitation. The completion of the VSD closure is accomplished by closing the atrium. Transesophageal echocardiography (TEE) is very important to assure the integrity of the repair after the closure by evaluating for residual intracardiac left-to-right shunting.

The exposure of doubly commited VSD is usually accomplished through a vertical incision in the pul-

\section{Figure 2. Left ventricolutomy for muscular VSD closure}

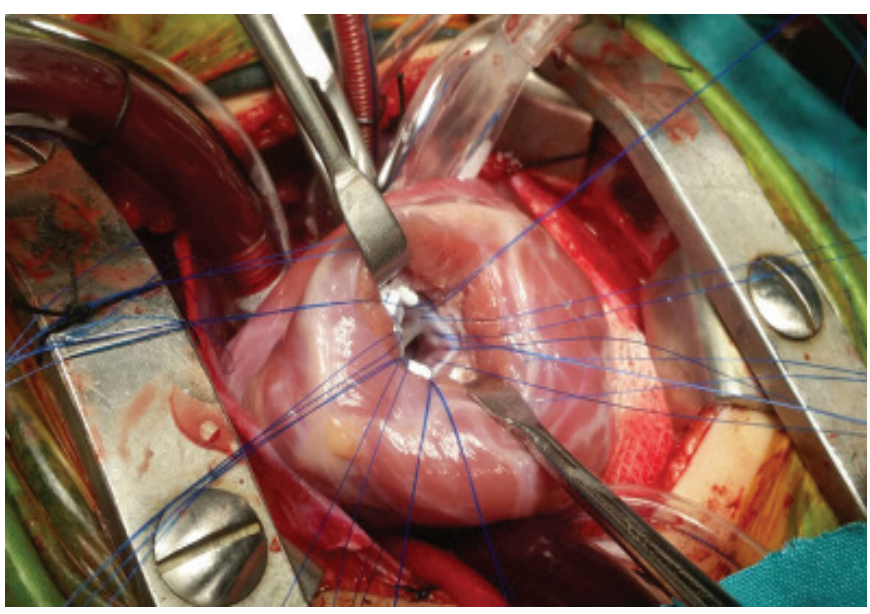


monary artery. Pulmonary valve is gently retracted. A critical part of the closure involves placing sutures directly in the base of the pulmonary valve cusp. Pledgetted sutures are placed circumferencially around the perimeter of the VSD.

Certain muscular VSDs (Swiss cheese) may be easier to patch on the left ventricular side because of the relatively smooth septum. Left ventricular incisions, however is associated with significant long-term ventricular dysfunction and should be avoided whenever possible (Figure-2). Multiple anterior muscular defects may be closed through short vertical right ventriculotomy. The defects are sandwitched between two strips of felt or pericardium, one placed inside and the other outside the right ventricle paralel to the left anterior descending coronary artery. Interrupted horizontal matress sutures are used. Midmuscular septal defects may be closed using a single composite patch to avoid injury to the conduction tissue. . $^{(2,6,10,11,12)}$

If the PVR is moderately elevated: (1) partial closure of the communication; (2) leaving a small ASD open while repairing posttricuspid defects; (3) placing a band on the pulmonary artery should be considered.

Over the past decade or so, transcatheter techniques for closure of ventricular septal defects have been developed. These methods have been especially useful for muscular defects, which can be the most difficult to Access surgically. Much interest has been generated in development of transcatheter approaches to close perimembranous defects. At present, this technique is not undertaken in most units because of the unacceptably high rate of post-procedure heart block associated with currently available devices. ${ }^{(13)}$

\section{Complications}

Premature rate death occurs in less than $2.5 \%$ of patients.when pulmonary resistence is low preoperatively. Repair of VSD during the first 1 or 2 years of life is curative for most patients, resulting in full functional activity and normal or near-normal life expectancy. Complications of surgical closure of VSD are generally related to injury to the anatomic structures, inadequate exposure and cardiopulmonary bypass.

Conduction system and the leaflets of tricuspid and aortic valves are at risk during VSD closure. Transient arrhythmias may be seen. Right bundle branch block (RBBB) is present late postoperatively in many patients in whom VSDs are repaired via right ventriculotomy. RBBB is less prevalent when the right atrial approach is used for VSD repair. Serious ventricular arrhythmias and sudden death late after repair of VSDs have been rare. Complete heart block requiring a pacemaker has been reported in 1-2 \% of cases. Prevalence is slightly higher in patients undergoing repair of multiple VSDs. Inlet VSDs extending posteriorly to the crux, associated with straddling tricuspid valve, also have increased prevalence of heart block after repair.

During suture placement, aortic valve injury can ocur and cause aortic insufficiency. Perimembranous VSD (rather than VSDs in the right ventricular outlet) and older age at operation contribute to presence of important aortic regurgitation after repair. VSD patch may rarely cause hemolysis. Tricuspid insuffiency can ocur if the leaflets are retracted inappropriately or after tricuspid detacment for exposure. In experienced centers, reoperation for residual VSD should be $2 \%$ or less. Late postoperative cardiac function is essentially normal when repair is done during the first 2 years of life by modern techniques through the right atrium..$^{(2,10,14)}$ 


\section{References}

1. Rubin AE, Lewin MB. Ventricular septal defect. In Moss \& Adams' Heart Disease in Infants, Children, and Adolescents, Including the Fetus and Young Adult. Eighth edition.Editors: Allen HD, Driscoll DJ, Shaddy RE, Feltes TF. 2013. p. 713-722

2. Mavroudis C, Backer CL, Jacobs JP, Anderson RH. Ventricular septal defect. In Pediatric Cardiac Surgery. Fourth edition. Editors: Mavroudis C, Backer CL. 2013. p. 311-336.

3. Chaudhry TA, Younas M, Baig A. Ventricular Septal defect and associated complications. JPMA 2011;61(10):1001-4.

4. Van Praagh R, Geva T, Kreutzer J. Ventricular septal defects: how shall we describe, name and classify them? J Am Coll Cardiol 1989; 14:1298.

5. Up to date, 2015

6. Knott-Craig CJ. Ventricular septal defect. In Mastery of Cardiothoracic Surgery. Second edition. Editors: Kaiser LR, Kron IL, Spray TL. 2007. p.750-758.

7. Lopes AA, Rabinovitch M. Statements on the management of pulmonary hypertension associated with congenital heart disease. Cardiol Young 2009(Suppl. S1):1-53.

8. Beghetti M, Galie` N, Bonnet D. Can "inoperable" congenital heart de- fects become operable in patients with pulmonary arterial hypertension? dream or reality? Congenital Heart Dis 2012;7:3-11.

9. Lopes AA, Barst RJ, Haworth SG, et al. Repair of congenital heart disease with associated pulmonary hypertension in children: what are the minimal investigative procedures? Consensus statement from the Congenital Heart Disease and Pediatric Task Forces, Pulmonary Vascular Research Institute (PVRI) Pulm Circ 2014;4(2):330-341. DOI: 10.1086/675995.

10. van Doorn C, de Leval MR. Surgery for congenital heart defects. Third edition. Editors: Stark J, De Leval M, Tsang VT. 2006. p.355-371.

11. Ventricular septal defect. In Comprehensive Surgical Management of Congenital Heart Disease. Second edition. Editor: Jonas RA. 2014. p.331345.

12. Ventricular septal defect. In Cardiac Surgery Safeguards and Pitfalls In Operative Technique.Fopurth edition. Editors Khonsari S, Sintek CF. 2008. p. 256-265.

13. Penny DJ, Vick GW. Ventricular septal defect. Lancet 2011; 377: 1103 12.

14. Ventricular septal defect. In Kirklin/Barrat-Boyes Cardiac Surgery. Fourth edition. Editors: Kouchoukos NT, Blackstone EH,Hanley FL, Kirklin J. 2013. p. $1275-1319$

Received: $16 / 11 / 2016$

Accepted: 21/03/2017

Published: 15/06/2017

Disclosure and conflicts of interest:

Conflicts of interest were not reported.

\section{Corresponding author:}

Prof. Dr. Zeynep Eyileten

Mail: zeyileten@gmail.com 